\title{
Schulkinder besuchen die Hebammen Ein Präventionsprojekt
}

\author{
Sandra Grieder, Hebamme FH, MSc Midwifery; Katherina Albert, Hebamme FH, MSc Midwifery; \\ Therese Hailer, Hebamme FH, MSc Midwifery \\ Zürcher Hochschule für Angewandte Wissenschaften (ZHAW), Winterthur
}

\section{Hintergrund}

Für den Unterricht in Grundschulen zu den Themen Schwangerschaft, Geburt und Wochenbett sind Hebammen gefragte Expertinnen (Schneider, 2008). Das Institut für Hebammen ist dabei, sein Angebot für Grundschulen auszuweiten und Unterrichtseinheiten in den hochschuleigenen Skillsräumen anzubieten. Zur Anwendung kommen hierbei die realitätsnahen und altersgerechten Simulationsmodelle.

\section{Projektziel}

Durch das Kennenlernen der Hebamme und ihres Arbeitsfeldes sollen Kinder der Mittelstufe möglichst früh erfahren, dass Schwangerschaft, Geburt und Wochenbett primär gesunde und natürliche Prozesse sind (SHV, 2007).

\section{Methode}

Die didaktische Gestaltung der Unterrichtseinheiten basiert auf Grundsätzen eines kompetenzorientierten und altersgerechten Unterrichts, wie Storyline-Methode, Mini-Simulationen und explorativem Lernen (Bachmann, 2013).

\section{Pilotunterricht}

Im Rahmen von zwei Unterrichtslektionen für eine Schulklasse aus Winterthur wurden die Themen Schwangerschaft, Geburt und Mutterschaft behandelt. Das Interesse der Mädchen und Knaben der 5. Klasse war sehr gross. Dies zeigte sich durch ihre aktive Teilnahme an Gesprächen über die eigene Geburt, das Einbringen am Workshop «Ein Kind entsteht» und das Mitwirken an der Mini-Simulation «Die Geburt».

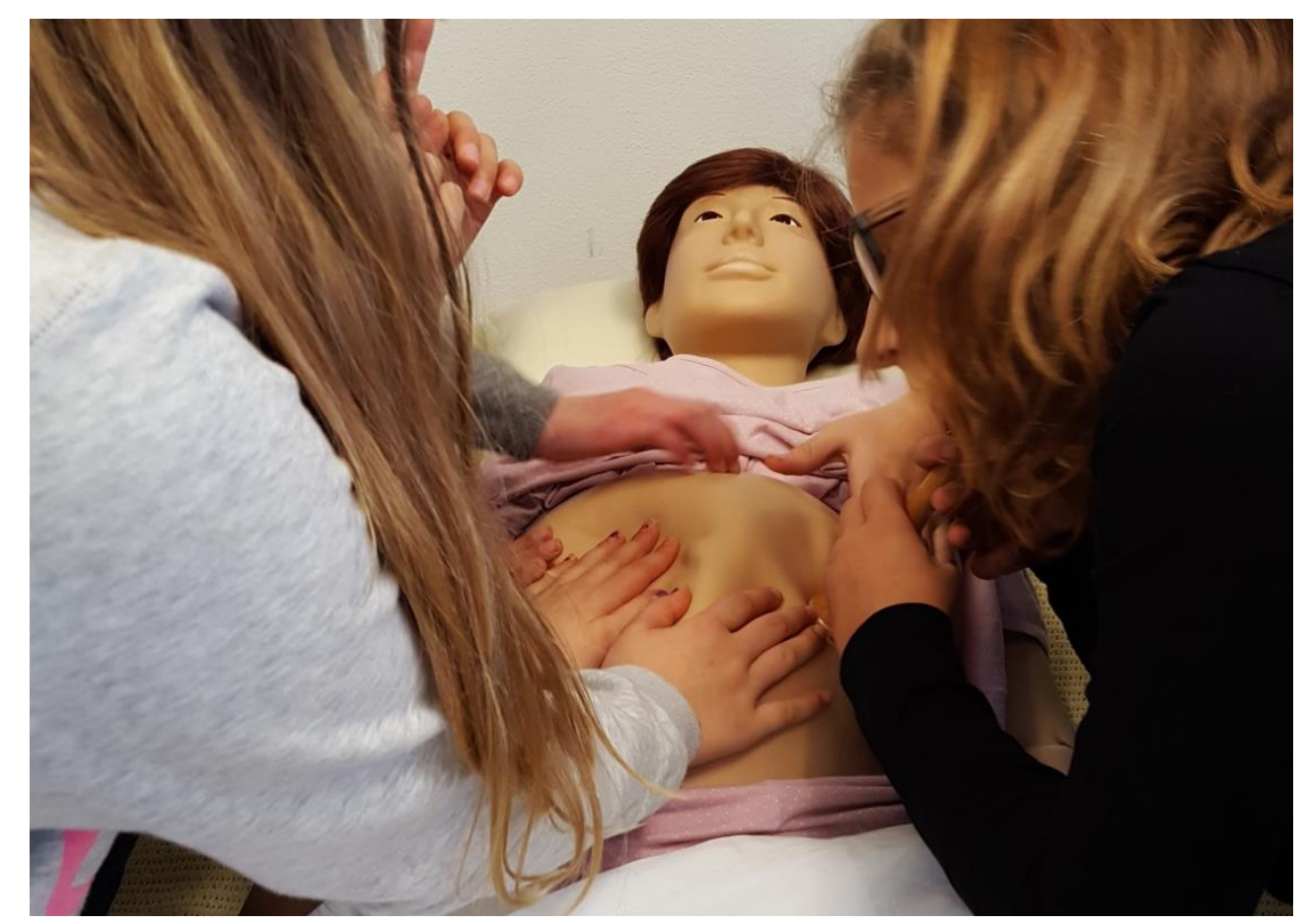

\section{Evaluation des Pilotunterrichts}

Die Unterrichtseinheit wurde in einem mehrstufigen Verfahren evaluiert.

Die Klassenlehrperson empfand die Lektionen als stufengerecht, abwechslungsreich und gut rhythmisiert: «Es kamen für mich nie Zweifel auf, dass es sich bei der Thematik um natürliche Prozesse im Lebenszyklus handelt. Ich würde den Unterricht wieder besuchen und ihn unbedingt weiterempfehlen.» Die Fragen und das Verhalten der Kinder während des Unterrichts lassen für die Dozentinnen den Rückschluss zu, dass die in den Lektionen behandelten Themen nachhaltig Einfluss auf eine weitere Auseinandersetzung mit der Thematik haben werden.

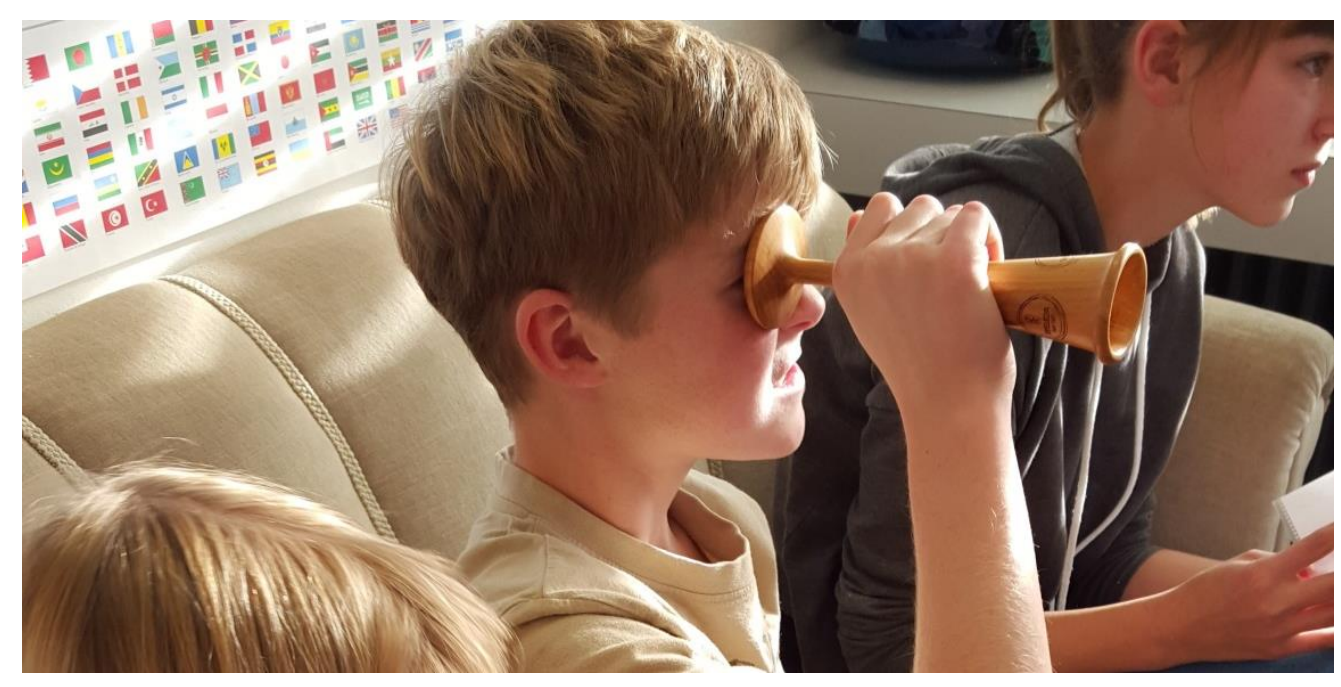

Ausblick

Das Rekrutierungsverfahren für einen erweiterten Pilotunterricht läuft. Der Einbezug von Studentinnen des sechsten Semesters des BSc Studiengangs Hebamme ist geplant. In diesem Zusammenhang wird auch eine mögliche fixe Einbettung ins Curriculum geprüft. Weitere Optionen, wie eine institutsübergreifende Zusammenarbeit oder ein Angebot im Sinne von «Train the Trainer» sind angedacht.

Referenzen

- Bachmann, H., \& PHZH (2013). Hochschullehre variantenreich gestalten: Kompetenzorientierte Hochschullehre. Bern: hep.

- Schneider, E. (2008). Bücher für Hebammen: Hebammen an Schulen. Frankfurt am Main: Mabuse-Verlag.

- SHV (2007). Berufsdefinition. Heruntergeladen von: www.hebamme.ch am 24.4.17

Kontakt:

Institut für Hebammen, ZHAW Gesundheit, Technikumstrasse 71, 8401 Winterthur Telefon 0589346474 / ihb.gesundheit@zhaw.ch www.zhaw.ch/gesundheit 